\title{
Sequential Multisine Excitation Signals for System Identification of Large Space Structures
}

\author{
Jesse B. Hoagg ${ }^{1}$, Seth L. Lacy ${ }^{2}$, Vit Babuška ${ }^{3}$, and Dennis S. Bernstein ${ }^{4}$
}

\begin{abstract}
In this paper, we present a novel sequence of multisine excitation signals that are more time efficient than sine dwell excitation while eliminating the effects of nonlinearity induced harmonics from the frequency response data. The sequential multisine excitation signal is demonstrated on the Deployable Optical Telescope testbed at Air Force Research Laboratory.
\end{abstract}

\section{INTRODUCTION}

Large space structures often require vibration suppression to meet performance requirements. Active control can be used to achieve high levels of disturbance rejection. However, many active control methods require high fidelity linear dynamic models that approximate the underlying dynamics. In addition, high fidelity models are required for performance prediction. Identifying linear dynamic models of large space structures can be difficult because large space structures are lightly damped, have dense modal spacing, have large dynamic range, respond to disturbances over a large frequency bandwidth, and may exhibit nonlinear responses.

There are numerous excitation signals used for linear system identification in the frequency domain, including random noise, burst random noise, pulse-impact, multisine, and sine dwell (also called stepped sine) [1-5]. Many excitation signals used to collect frequency response data are poorly suited to systems that exhibit nonlinear responses. Specifically, the nonlinear responses to these excitations create undesired artifacts in the frequency response data. Furthermore, these undesired artifacts are indistinguishable from the linear frequency response and can significantly impact the characteristics of a linear model identified using frequency domain system identification methods.

In particular, it is difficult to use broadband excitation signals to obtain linear frequency response data for a system that exhibits nonlinear distortions due to harmonics. In $[2,3]$, it is suggested that a multisine excitation signal may be designed to reduce or eliminate nonlinear distortions. However, this method requires certain knowledge of the system's nonlinearities, that is, we must know the harmonics that are being excited. In addition, the method suggested in $[2,3]$ is difficult to implement when the system has dense modal spacing and/or there are many base tones for which higher-order harmonics are being excited. Therefore, sine dwell excitation signals are often used to eliminate nonlinear distortions. However, sine dwell testing can be much less time efficient than broadband excitation signals [4]. In practice, sine dwell testing is often abbreviated, resulting in lower quality data.

In the present paper, we consider a novel sequence of multisine excitation signals designed to obtain system identification data over a large frequency bandwidth, while eliminating nonlinearity induced harmonics from the frequency response data. Furthermore, the method presented in this paper is more time efficient than sine dwell testing.

\section{Problem Formulation}

The goal of frequency domain system identification is to obtain a linear time-invariant model that approximates the dynamics

\footnotetext{
${ }^{1}$ National Defense Science and Engineering Graduate Fellow, Department of Aerospace Engineering, The University of Michigan

${ }^{2}$ Air Force Research Laboratory, Space Vehicles Directorate, Kirtland AFB, NM

${ }^{3}$ General Dynamics AIS

${ }^{4}$ Professor, Department of Aerospace Engineering, The University of Michigan
}

of an unknown plant. In general, the plant may have nonlinear time-varying dynamics. Plant nonlinearities often induce harmonic responses to sinusoidal input signals. Consider, for example, the nonlinear system shown in Figure 1, which has asymptotically stable linear dynamics $\mathcal{L}$ with a static input nonlinearity $\mathcal{N}_{1}$ and a static output nonlinearity $\mathcal{N}_{2}$. The nonlinearities $\mathcal{N}_{1}$ and $\mathcal{N}_{2}$ of this system can induce harmonic responses. In particular, if $\mathcal{N}_{1}$ and $\mathcal{N}_{2}$ are analytic functions, then the nonlinearities can only induce integer superharmonics. If $\mathcal{N}_{1}$ and $\mathcal{N}_{2}$ are analytic functions, then they can be approximated to arbitrary accuracy by polynomials, that is,

$$
\begin{aligned}
& \mathcal{N}_{1}(x)=\alpha_{0}+\alpha_{1} x+\cdots+\alpha_{n-1} x^{n-1}+\alpha_{n} x^{n}+\cdots, \\
& \mathcal{N}_{2}(x)=\beta_{0}+\beta_{1} x+\cdots+\beta_{n-1} x^{n-1}+\beta_{n} x^{n}+\cdots
\end{aligned}
$$

where the real coefficients $\alpha_{0}, \alpha_{1}, \ldots$ and $\beta_{0}, \beta_{1}, \ldots$ are determined by the Taylor series expansions of $\mathcal{N}_{1}$ and $\mathcal{N}_{2}$, respectively. Now, we assume that the system input is the sinusoid $u(t)=$ $C_{1} \sin (2 \pi \omega t)+C_{2} \cos (2 \pi \omega t)$. Then the input to the linear dynamical system $\mathcal{L}$ is $u_{\mathcal{L}}(t)=\mathcal{N}_{1}(u(t))$. Using trigonometric identities, $u_{\mathcal{L}}(t)$ may be expressed as a sum of sinusoids with frequencies $\omega, 2 \omega, 3 \omega, \ldots$. Since $\mathcal{L}$ is an asymptotically stable linear system and $u_{\mathcal{L}}(t)$ is a sum of sinusoids with frequencies $\omega, 2 \omega, 3 \omega, \ldots$, it follows that the output $y_{\mathcal{L}}(t)=\mathcal{L}\left(\mathcal{N}_{1}(u(t))\right)$ at steady state is a sum of sinusoids with frequencies $\omega, 2 \omega, 3 \omega, \ldots$. Since $y_{\mathcal{L}}(t)$ is a sum of harmonically related sinusoids, we use the Taylor series expansion of $\mathcal{N}_{2}(x)$ and trigonometric identities to express $y(t)=\mathcal{N}_{2}\left(y_{\mathcal{L}}(t)\right)$ as a sum of sinusoids with frequencies $\omega, 2 \omega, 3 \omega, \ldots$. Therefore, the nonlinearities $\mathcal{N}_{1}$ and $\mathcal{N}_{2}$ can induce integer superharmonic responses.

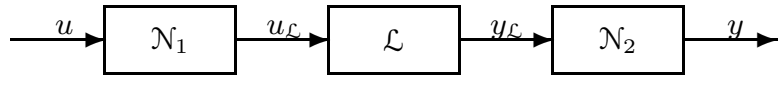

Fig. 1. Linear dynamic system with static input and output nonlinearities.

In general, a system may have nonlinear time-varying dynamics that do not possess the block structure shown in Figure 1. In the present paper, we consider plants with nonlinear time-invariant dynamics and make the following assumptions.

(A1) The plant has a globally asymptotically stable equilibrium.

(A2) The plant is linearizable about the equilibrium.

(A3) The plant nonlinearities can induce only integer subharmonic and superharmonic responses of a base tone.

The objective of this paper is to design an excitation signal to obtain frequency response data for frequency domain system identification. We require that the excitation signal satisfies the following conditions.

(i) The signal must excite the frequency band $\Omega \triangleq$ $\left[\omega_{\min }, \omega_{\max }\right]$, where $0<\omega_{\min }<\omega_{\max }$.

(ii) The signal must be sufficiently rich to excite frequencies on a discrete grid with spacing less than or equal to $\eta$ where $0<\eta \leq \omega_{\min }$.

(iii) No leakage in the Fourier Transform of the excitation signal and output signal.

(iv) The base tone response of the plant must be distinguishable from nonlinear distortions caused by subharmonics and superharmonics. 
Without loss of generality, we assume that $\frac{\omega_{\min }}{\eta}$ and $\frac{\omega_{\max }}{\eta}$ are integers.

In addition to conditions (i)-(iv), we seek an input signal that does not require a long test time. Certain input signals can require long test times to meet conditions (i)-(iv). In fact, test times can be too long for practical implementation. The purpose of this paper is to design an excitation signal that satisfies $(i)-(i v)$, while requiring test times short enough for practical implementation.

In the following sections, we examine the properties of several common excitation signals. Specifically, we consider random noise, burst random noise, pulse-impact, multisine, and sine dwell excitation signals. For more information on these input signals, see Chapter 4 of [5]. We demonstrate that none of these signals satisfies conditions (i)-(iv), while being practically implementable with respect to test time.

\section{RANDOM NoISE, Burst RANDOM NoIse, AND PULSE-IMPACT}

Random noise excitation requires a test time $T_{\mathrm{r}} \geq \frac{1}{\omega_{\min }}$ to achieve the desired frequency resolution and bandwidth. Test times $T_{\mathrm{r}}>>\frac{1}{\omega_{\min }}$ are often required to infuse the frequency band $\Omega$ with sufficient power, to obtain a satisfactory signal-to-noise ratio, and to satisfy condition (ii).

Random noise signals suffer from leakage since they are aperiodic. Leakage can be alleviated by windowing the input and output data or by repeatedly applying an input signal. Alternatively, leakage can be addressed by using burst random noise or pulse-impact excitation signals. A burst random noise signal is $u_{\mathrm{br}}(t)=w(t) d(t)$, where $w(t)$ is a random variable and $d(t)=\left\{\begin{array}{cc}1, & 0 \leq t<T_{1} \\ 0, & T_{1} \leq t \leq T_{\mathrm{r}}\end{array}\right.$. A pulse-impact signal is $u_{\mathrm{pi}}(t)=$ $\left\{\begin{array}{cc}A_{\mathrm{pi}}, & 0 \leq t<T_{1} \\ 0, & T_{1} \leq t<T_{\mathrm{r}}\end{array}\right.$, where $A_{\mathrm{pi}} \in \mathbb{R}$. If the test time $T_{\mathrm{r}}$ is long enough so that the plant's response decays to a negligible amount by time $T_{\mathrm{r}}$, then leakage errors will be negligible [5]. However, for lightly damped systems with low frequency modes, the plant's response can take a long time to decay. In practice, windowing is often used with both burst random noise and pulseimpact excitation signals to allow for shorter test times.

To make matters worse, random and pulse excitation signals do not provide insight into the plant's nonlinearities. Specifically, the plant's linear response is indistinguishable from nonlinear distortions since random noise and pulse signals simultaneously excite the entire bandwidth of interest. Thus random noise, burst random noise, and pulse-impact signals do not satisfy condition (iv).

\section{Multisine}

A multisine signal is a summation of sinusoids with varying frequencies and phases. A Schroeder-phased multisine is the sum of sinusoids where the phases are selected to obtain low peak-topeak amplitudes [1]. Define $N \triangleq \frac{\omega_{\max }-\omega_{\min }}{\eta}+1$ and consider the Schroeder-phased multisine

$$
u_{\mathrm{m}}(t)=\sum_{k=1}^{N} A_{\mathrm{m}} \cos \left(2 \pi \omega_{k} t+\phi_{k}\right),
$$

where $A_{\mathrm{m}} \in \mathbb{R}$ and, for $k=1, \ldots, N, \phi_{k} \triangleq \frac{-k(k-1) \pi}{N}$ and $\omega_{k} \triangleq \omega_{\min }+\eta(k-1)$. The Schroeder phases $\phi_{1}, \ldots, \phi_{N}$ have been used for simplicity. However, the phases may be selected to obtain other desired properties of the input signal. For example, the phases may be selected to minimize the crest-factor [6]. Note that the multisine (4.1) has a flat power spectrum over $\Omega$.
The multisine is periodic with period $\frac{1}{\eta}$. Leakage is eliminated if

$$
T_{\mathrm{m}}=l_{\mathrm{m}} \frac{1}{\eta}+\Delta t_{\mathrm{m}}
$$

where $l_{\mathrm{m}} \in \mathbb{N}$ is the number of periods used for averaging and $\Delta t_{\mathrm{m}}>0$ is the time required for the system's response to $u_{\mathrm{m}}(t)$ to converge to steady state. Multisines are efficient with respect to test time since they simultaneously excite the entire frequency band $\Omega$ and require only one settling period $\Delta t_{\mathrm{m}}$.

Simultaneous excitation of the entire bandwidth $\Omega$ is undesirable for dealing with nonlinearities. It is impossible to separate the linear response from harmonic responses caused by plant nonlinearities, and thus multisine excitation signals do not satisfy condition (iv).

5. SINE DWELL

A sine dwell excitation signal is a sequence of sinusoidal excitation signals, each with different frequency. Define $N \triangleq$ $\frac{\omega_{\max }-\omega_{\min }}{\eta}+1$ and, for $k=1, \ldots, N$, consider the sinusoids $u_{\mathrm{s}, k}(t)=A_{\mathrm{s}, k} \sin \left(2 \pi \omega_{k} t\right)$,

where for $k=1, \ldots, N, A_{\mathrm{s}, k} \in \mathbb{R}$ and $\omega_{k} \triangleq \omega_{\min }+\eta(k-1)$. Sine dwell testing is performed by conducting the sequence of $N$ sinusoidal inputs $u_{\mathrm{s}, 1}(t), \ldots, u_{\mathrm{s}, N}(t)$. This populates the frequency band $\Omega$ with frequency spacing $\eta$.

The sinusoids $u_{\mathrm{s}, 1}(t), \ldots, u_{\mathrm{s}, N}(t)$ are periodic with periods $\frac{1}{\omega_{1}}, \ldots, \frac{1}{\omega_{N}}$, respectively. For $k=1, \ldots, N$, let $T_{\mathrm{s}, k}$ be the measurement time associated with $u_{\mathrm{s}, k}(t)$. Leakage is eliminated by letting $T_{\mathrm{s}, k}=l_{\mathrm{s}, k} \frac{1}{\omega_{i}}$ where, for $k=1, \ldots, N, l_{\mathrm{s}, k} \in \mathbb{N}$ is the number of periods used for averaging after the steady state response has been achieved.

Unlike the other excitation signals we have discussed thus far, a sine dwell excitation signal allows the harmonic response to be separated from the base tone response. For $k=1, \ldots, N$, the sinusoid $u_{\mathrm{s}, k}(t)$ is used to estimate the base tone response at frequency $\omega_{k}$, but the harmonic response can be distinguished from the base tone response since the harmonic responses due to nonlinearities occur at frequencies other than $\omega_{k}$. In fact, sine dwell testing provides detailed information of nonlinear distortions.

The major drawback to sine dwell testing is lengthy test time. The test time required for sine dwell is

$$
T_{\mathrm{s}}=\sum_{k=1}^{N} T_{\mathrm{s}, k}+\Delta t_{\mathrm{s}, k}=\sum_{k=1}^{N} l_{\mathrm{s}, k} \frac{1}{\omega_{k}}+\Delta t_{\mathrm{s}, k},
$$

where, for $k=1, \ldots, N, \Delta t_{\mathrm{s}, k}>0$ is the time required for the system's response to the sinusoid $u_{\mathrm{s}, k}(t)$ to settle. Sine dwell testing can require significantly longer test times than multisine testing.

\section{Test Times for Multisine And SINE DWELL EXCITATION}

In this section, we compare the test times required for multisine and sine dwell excitation signals. Specifically, we compare the test times required to obtain frequency response data with equivalent signal-to-noise ratios. The quality of system identification data is most accurately described by the signal-to-noise ratios of the input and output signals. Input signals are usually assumed to be noisefree, and the output signal-to-noise ratio depends on the output noise, the input power spectral density, and the system dynamics. If the system is linear, then the output power spectral density is the product of the input power spectral density and the magnitude of the system's transfer function. Although this paper considers nonlinear systems, if input amplitudes are small, then the output power spectral density may be approximated by the product of 
input power spectral density and the magnitude of the linearized transfer function. We use the signal-to-noise ratio obtained from the approximate output power spectral density as a measure of data quality.

Let $F_{s}$ denote the sampling frequency of the multisine and sine dwell excitation signals. The input power spectral density for one period of the multisine $u_{\mathrm{m}}(t)$ is $U_{\mathrm{m}}(k)=\sqrt{\frac{F_{s}}{\eta}} \frac{A_{\mathrm{m}}}{2}$, and the input power spectral density for one period of each sine dwell $u_{\mathrm{s}, 1}(t), \ldots, u_{\mathrm{s}, N}(t)$ is $U_{\mathrm{s}}(k)=\sqrt{\frac{F_{s}}{\omega_{k}}} \frac{A_{\mathrm{s}, k}}{2}$.

Let $G(z)$ be the discrete transfer function matrix of the linearized and sampled system. Then the approximate output power spectral densities obtained from multisine and sine dwell excitation are

$$
\begin{gathered}
Y_{\mathrm{m}}(k) \triangleq\left\|G\left(e^{\jmath \omega_{k}}\right)\right\| U_{\mathrm{m}}(k)=\sqrt{\frac{F_{s}}{\eta}} \frac{A_{\mathrm{m}}}{2}\left\|G\left(e^{\jmath \omega_{k}}\right)\right\|, \\
Y_{\mathrm{s}}(k) \triangleq\left\|G\left(e^{\jmath \omega_{k}}\right)\right\| U_{\mathrm{s}}(k)=\sqrt{\frac{F_{s}}{\omega_{k}}} \frac{A_{\mathrm{s}, k}}{2}\left\|G\left(e^{\jmath \omega_{k}}\right)\right\|,
\end{gathered}
$$

respectively.

Input amplitudes for the mutlisine and sine dwell excitation signals must be normalized to compare testing times, since linear approximations are only valid for small amplitudes and actuator saturation imposes practical limitations. If we design multisine and sine dwell excitation signals such that $A_{\mathrm{m}}=A_{\mathrm{s}, 1}=\cdots=$ $A_{\mathrm{s}, N}=A$ where $A>0$, then the excitation signals will not have equal peak values. Numerical results indicate that the peak value of the Schroeder-phased multisine (4.1) is proportional to $\sqrt{N}$. Furthermore, the constant of proportionality is independent of the frequency spacing $\eta$.

Let $A_{\mathrm{s}, 1}=\cdots=A_{\mathrm{s}, N}=A$ and $A_{\mathrm{m}}=\frac{A}{\sqrt{N}}$, where $A>0$ is the desired peak signal value. We use the normalization $A_{\mathrm{m}}=$ $\frac{A}{\sqrt{N}}$, which is convenient for the subsequent analysis, even though the constant of proportionality between $\bar{u}_{\mathrm{m}}$ and $\sqrt{N}$ is not exactly one. The approximate output power spectral densities satisfy

$$
\begin{aligned}
Y_{\mathrm{m}}(k) & =\sqrt{\frac{F_{s}}{\eta}} \frac{A}{2 \sqrt{N}}\left\|G\left(e^{\jmath \omega_{k}}\right)\right\|, \\
Y_{\mathrm{s}}(k) & =\sqrt{\frac{F_{s}}{\omega_{k}}} \frac{A}{2}\left\|G\left(e^{\jmath \omega_{k}}\right)\right\| .
\end{aligned}
$$

Let $N_{y}(k)$ denote the discrete Fourier transform of the output noise, then the output signal-to-noise ratio for a single period of the multisine excitation $u_{\mathrm{m}}(t)$ is

$$
\frac{Y_{\mathrm{m}}(k)}{N_{y}(k)}=\frac{1}{\sqrt{\eta N}} \frac{A \sqrt{F_{s}}}{2 N_{y}(k)}\left\|G\left(e^{\jmath \omega_{k}}\right)\right\|,
$$

and the output signal-to-noise ratio for an excitation signal with a single period of each sine dwell $u_{\mathrm{s}, 1}(t), \ldots, u_{\mathrm{s}, N}(t)$ is

$$
\frac{Y_{\mathrm{s}}(k)}{N_{y}(k)}=\frac{1}{\sqrt{\omega_{k}}} \frac{A \sqrt{F_{s}}}{2 N_{y}(k)}\left\|G\left(e^{\jmath \omega_{k}}\right)\right\| .
$$

The test times required for the multisine and sine dwell excitations are given by (4.2) and (5.2), respectively. The number of multisine periods $l_{\mathrm{m}}$ and sine dwell periods $l_{\mathrm{s}, 1}, \ldots, l_{\mathrm{s}, N}$ used for averaging should be chosen to ensure that the output signals have comparable signal-to-noise ratios.

We assume that $N_{y}(k)$ is filtered white noise, independent of both $Y_{\mathrm{m}}(k)$ and $Y_{\mathrm{s}}(k)$, with a finite second moment. Under these mild assumptions, averaging the output data over $l \in \mathbb{N}$ periods reduces output uncertainty proportionally to $\frac{1}{\sqrt{l}}$ [5]. Let $\mathrm{SNR}_{\mathrm{m}}(k)$ denote the output signal-to-noise ratio of the multisine excitation data when $l_{\mathrm{m}}$ averages are performed, and let $\operatorname{SNR}_{\mathrm{s}}(k)$ denote the output signal-to-noise ratio of the sine dwell excitation data when $l_{\mathrm{s}, 1}, \ldots, l_{\mathrm{s}, N}$ averages are performed. Therefore, the signalto-noise ratios satisfy

$$
\begin{aligned}
\operatorname{SNR}_{\mathrm{m}}(k) & \propto \sqrt{l_{\mathrm{m}}} \frac{1}{\sqrt{\eta N}} \frac{A \sqrt{F_{s}}}{2 N_{y}(k)}\left\|G\left(e^{\jmath \omega_{k}}\right)\right\|, \\
\operatorname{SNR}_{\mathrm{s}}(k) & \propto \sqrt{l_{\mathrm{s}, k}} \frac{1}{\sqrt{\omega_{k}}} \frac{A \sqrt{F_{s}}}{2 N_{y}(k)}\left\|G\left(e^{\jmath \omega_{k}}\right)\right\| .
\end{aligned}
$$

If $l_{\mathrm{s}, k}=l \frac{\omega_{k}}{\eta}$ and $l_{\mathrm{m}}=l N$, then it follows from (6.7) and (6.8) that the output signals obtained from the multisine and sine dwell excitations have comparable signal-to-noise ratios. To obtain these comparable signal-to-noise levels, the multisine excitation signal requires

$$
T_{\mathrm{m}}=l_{\mathrm{m}} \frac{1}{\eta}+\Delta t_{\mathrm{m}}=N \frac{l}{\eta}+\Delta t_{\mathrm{m}}
$$

and sine dwell excitation requires

$$
T_{\mathrm{s}}=\sum_{k=1}^{N} l_{\mathrm{s}, k} \frac{1}{\omega_{k}}+\Delta t_{\mathrm{s}, k}=N \frac{l}{\eta}+\sum_{k=1}^{N} \Delta t_{\mathrm{s}, k} .
$$

It follows from (6.9) and (6.10) that sine dwell testing can take significantly longer than multisine testing. Specifically, sine dwell testing requires additional time for the system to settle each time the sine dwell frequency is changed. For linear systems, practical settling times $\Delta t_{\mathrm{m}}$ and $\Delta t_{\mathrm{s}, 1}, \ldots, \Delta t_{\mathrm{s}, N}$ can be estimated from the system's time constant. Furthermore, the time constant of a linear system is independent of input signal. Therefore, in practice, it is often assumed that settling time is independent of the harmonic content of the excitation signal.

If we assume that the settling times $\Delta t_{\mathrm{m}}$ and $\Delta t_{\mathrm{s}, 1}, \ldots, \Delta t_{\mathrm{s}, N}$ are independent of excitation signal, then sine dwell testing requires $(N-1) \Delta t_{\mathrm{m}}$ time units longer than multisine testing. If, in addition, the frequency band of interest is large and/or the frequency spacing is small, then $N$ will be large and sine dwell testing will take significantly longer than multisine testing. Furthermore, if the system is lightly damped, then settling times can be very long, exacerbating the disparity in test times.

For example, consider a single-input system and design input signals to excite frequencies from $\omega_{\min }=0.1 \mathrm{~Hz}$ to $\omega_{\max }=100$ $\mathrm{Hz}$ with a frequency spacing of $\eta=0.1 \mathrm{~Hz}$. Assume that the system requires 5 seconds to settle. The multisine signal (4.1) requires $T_{\mathrm{m}}=N \frac{l}{\eta}+\Delta t_{\mathrm{m}}=10,000 l+5 \mathrm{sec}$, and the sine dwell signal (5.1) requires $T_{\mathrm{s}, k}=N \frac{l}{\eta}+\sum_{k=1}^{N} \Delta t_{\mathrm{s}, k}=10,000 l+$ $5,000 \mathrm{sec}$. Thus, sine dwell testing requires an additional 4,995 seconds (or 1 hour 23 minutes 15 seconds). If $l$ is small, then 4,995 seconds constitutes a significant portion of the total testing time. Furthermore, if the frequency band $\Omega$ is increased or the frequency spacing $\eta$ is decreased, then the disparity between the sine dwell test time and the multisine test time will increase.

\section{Sequential Multisines}

In this section, we introduce a novel excitation signal consisting of a sequence of multisines that are designed to retain the benefits of sine dwell signals while being more time efficient. Specifically, we design a sequence of multisines that allow the linear response to be distinguished from nonlinear distortions.

Let $M \in \mathbb{N}$ be the least positive integer such that $M>$ $\log _{2} \frac{\omega_{\max }}{\omega_{\min }}$. In general, $M \leq N$ and if the bandwidth of interest is large and/or $\eta$ is small, then $M<<N$.

Divide the frequency band $\Omega$ into $M$ segments. For $i=$ $1, \ldots, M$, define the frequency bands $\Omega_{i} \triangleq\left[\bar{\omega}_{i}, \bar{\omega}_{i+1}\right)$, where, 
for $i=1, \ldots, M, \bar{\omega}_{i} \triangleq 2^{i-1} \omega_{\min }$, and $\bar{\omega}_{M+1} \triangleq \omega_{\max }+\eta$. Note that, for $i=1, \ldots, M, \Omega_{i}$ does not contain integer subharmonics or superharmonics of the base frequencies contained in $\Omega_{i}$, which is the salient feature of partitioning the frequency band.

Now, we consider a sequence of $M$ Schroeder-phased multisines. For $i=1, \ldots, M$, define $N_{i} \triangleq \frac{\bar{\omega}_{i+1}-\bar{\omega}_{i}}{\eta}$ and consider

$$
u_{\mathrm{sm}, i}(t)=\sum_{j=1}^{N_{i}} A_{\mathrm{sm}, i} \cos \left(2 \pi \omega_{i, j} t+\phi_{i, j}\right),
$$

where, for $i=1, \ldots, M, A_{\mathrm{sm}, i} \in \mathbb{R}$ and, for $j=1, \ldots, N_{i}$,

$$
\phi_{i, j} \triangleq \frac{-j(j-1) \pi}{N_{i}}, \quad \omega_{i, j} \triangleq \bar{\omega}_{i}+\eta(j-1) .
$$

If, for example, $\omega_{\min }=\eta$, then $N_{1}=1, N_{2}=2, N_{3}=4$, $N_{4}=8, \ldots, N_{M-1}=2^{M-2}$ and $N_{M}=2^{M-2}+\frac{\omega_{\max }}{\eta}+1$. Again, the Schroeder phases have been chosen for simplicity, but the phases may be chosen to satisfy other excitation signal criteria.

For $i=1, \ldots, M$, the multisine $u_{\mathrm{sm}, i}(t)$ excites frequencies in the band $\Omega_{i}$ but does not excite frequencies outside of the band $\Omega_{i}$. For $i=1, \ldots, M$, let $T_{\mathrm{sm}, i}$ be the measurement time associated with $u_{\mathrm{sm}, i}(t)$. For $i=1, \ldots, M$, letting $T_{\mathrm{sm}, i}=l_{\mathrm{sm}, i} \frac{1}{\eta}$ where $l_{\mathrm{sm}, i} \in \mathbb{N}$ eliminates leakage. Then the test time required for the sequential multisine signal is given by

$$
T_{\mathrm{sm}}=\sum_{i=1}^{M} T_{\mathrm{sm}, i}+\Delta t_{\mathrm{sm}, i}=\frac{1}{\eta} \sum_{i=1}^{M} l_{\mathrm{sm}, i}+\Delta t_{\mathrm{sm}, i}
$$

where, for $i=1, \ldots, M, \Delta t_{\mathrm{sm}, i}>0$ is the time required for the system's response to $u_{\mathrm{sm}, i}(t)$ to settle to harmonic steady state.

Now, we compare the sequential multisine test time with the multisine and sine dwell test times. For $i=1, \ldots, M$, let $A_{\mathrm{sm}, i}=\frac{A}{\sqrt{N_{i}}}$, thus normalizing the peak signal values to approximately $A>0$. The number of sequential multisine periods $l_{\mathrm{sm}, 1}, \ldots, l_{\mathrm{sm}, M}$ used for averaging must be chosen to ensure that the output signal obtained from the sequential multisine excitation has a signal-to-noise ratio $\operatorname{SNR}_{\mathrm{sm}}(k)$ comparable to those obtain from the multisine and sine dwell excitations. Performing the signal-to-noise analysis for the sequential multisine excitation (similar to the analysis of Section 6) yields that if, for $i=1, \ldots, M, l_{\mathrm{sm}, i}=l N_{i}$, then $\operatorname{SNR}_{\mathrm{sm}}(k)$ is comparable to $\mathrm{SNR}_{\mathrm{m}}(k)$ and $\mathrm{SNR}_{\mathrm{S}}(k)$. To obtain this comparable signal-tonoise level, the sequential multisine excitation requires

$$
T_{\mathrm{sm}}=\frac{1}{\eta} \sum_{i=1}^{M} l N_{i}+\Delta t_{\mathrm{sm}, i}=N \frac{l}{\eta}+\sum_{i=1}^{M} \Delta t_{\mathrm{sm}, i} .
$$

It follows from (6.9), (6.10), and (7.4) that sequential multisine testing is less time efficient than single multisine testing but more time efficient than sine dwell testing. In fact, if the system's settling times $\Delta t_{\mathrm{m}}, \Delta t_{\mathrm{s}, 1}, \ldots, \Delta t_{\mathrm{s}, N}$, and $\Delta t_{\mathrm{s}, 1}, \ldots, \Delta t_{\mathrm{s}, N}$ are equal, then sine dwell testing takes $(N-M) \Delta t_{\mathrm{m}}$ more time units than sequential multisine testing. Furthermore, $N$ increases quicker than $M$ as the number of discrete frequency points increases. Specifically, $N$ increases linearly with the number of discrete frequencies whereas $M$ is proportional to the logarithm of the number of discrete frequencies. Therefore, $N-M$ may be very large.

Consider again the example given in Section 6. The objective is to design an input signal that excites frequencies from $\omega_{\min }=0.1$ $\mathrm{Hz}$ to $\omega_{\max }=100 \mathrm{~Hz}$ with a discrete-frequency spacing of $\eta=$ $0.1 \mathrm{~Hz}$. We assume that for all harmonic excitations, the system requires 5 seconds to settle. Recall that the a single multisine requires a test time of $T_{\mathrm{m}}=10,000 \mathrm{l}+5 \mathrm{sec}$, and a sine dwell input requires a test time of $T_{\mathrm{s}}=10,000 l+5,000 \mathrm{sec}$. In contrast, the sequential multisine (7.1) requires a test time $T_{\mathrm{sm}}=N \frac{l}{\eta}+$ $\sum_{i=1}^{M} \Delta t_{\mathrm{sm}, i}=10,000 l+50 \mathrm{sec}$. Thus sine dwell excitation requires 4,950 seconds (or 1 hour 22 minutes 30 seconds) longer than sequential multisine excitation.

For $i=1, \ldots, M, u_{\mathrm{sm}, i}(t)$ is used to estimate the base tone response over the frequency band $\Omega_{i}$, but the harmonic response due to nonlinearities occur at frequencies not contained in the $\Omega_{i}$. The linear response over $\Omega_{i}$ is distinguishable from the nonlinear distortions that occur at frequencies outside of $\Omega_{i}$. Thus, the sequential multisine satisfies conditions (i)-(iv) while being less time consuming than sine dwell testing.

\section{Multi-Input Sequential Multisine Excitation}

In the previous sections, we designed multisine, sine dwell, and sequential multisine excitation signals assuming that the system is excited one input at a time, that is, data are collected by exciting input channel 1 , then by exciting input channel 2 , and continuing until the system has been excited through all input channels. Assume that the system has $p$ inputs. Then the test times given by (6.9), (6.10), and (7.4) are multiplied by $p$ to yield the total test time.

For a sine dwell excitation signal, exciting the system using only one input channel at a time is not time efficient. In fact, sine dwell testing can be performed by simultaneously exciting all input channels, and the test time for sine dwell excitation is independent of the number of inputs.

Similarly, sequential multisine excitation signals can be designed so that all input channels may be excited simultaneously, yielding a test time that is independent of $p$. We consider the frequency bands $\Omega_{1}, \ldots, \Omega_{M}$ given by (7.1), and defined the finer frequency spacing $\bar{\eta} \triangleq \frac{\eta}{p}$. For each input channel, we consider a sequence of $M$ Schroeder-phased multisines. For $i=1, \ldots, M$, consider

$$
\begin{aligned}
u_{\mathrm{sm}, i}^{1}(t) & =\sum_{j=1}^{N_{i}} A_{\mathrm{sm}, i} \cos \left(2 \pi\left(\omega_{i, j}\right) t+\phi_{i, j}\right), \\
u_{\mathrm{sm}, i}^{2}(t) & =\sum_{j=1}^{N_{i}} A_{\mathrm{sm}, i} \cos \left(2 \pi\left(\omega_{i, j}+\bar{\eta}\right) t+\phi_{i, j}\right), \\
\vdots & \\
u_{\mathrm{sm}, i}^{p}(t) & =\sum_{j=1}^{N_{i}} A_{\mathrm{sm}, i} \cos \left(2 \pi\left(\omega_{i, j}+(p-1) \bar{\eta}\right) t+\phi_{i, j}\right) .
\end{aligned}
$$

In this notation, input channel 1 is excited with the sequence of multisines $u_{\mathrm{sm}, 1}^{1}(t), \ldots, u_{\mathrm{sm}, M}^{1}(t)$, input channel 2 is excited with the sequence of multisines $u_{\mathrm{sm}, 1}^{2}(t), \ldots, u_{\mathrm{sm}, M}^{2}(t)$, and input channel $p$ is excited with the sequence of multisines $u_{\mathrm{sm}, 1}^{p}(t), \ldots, u_{\mathrm{sm}, M}^{p}(t)$.

For $i=1, \ldots, M$, the multisines $u_{\mathrm{sm}, i}^{1}(t), \ldots, u_{\mathrm{sm}, i}^{p}(t)$ are periodic with period $\frac{1}{\bar{\eta}}$. Therefore, leakage is eliminated by letting the test time associated with each multisine in the sequence be $\bar{T}_{\mathrm{sm}, i}=\bar{l}_{\mathrm{sm}, i} \frac{1}{\bar{\eta}}$ where $\bar{l}_{\mathrm{sm}, i} \in \mathbb{N}$. Since all input channels are simultaneously excited, the required test time is given by

$$
T_{\mathrm{sm}}=\frac{1}{\bar{\eta}} \sum_{i=1}^{M} \bar{l}_{\mathrm{sm}, i}+\bar{\Delta} t_{\mathrm{sm}, i}
$$


where, for $i=1, \ldots, M, \bar{\Delta} t_{\mathrm{sm}, i}$ is the time required for the system's response to $u_{\mathrm{sm}, i}^{1}(t), \ldots, u_{\mathrm{sm}, i}^{p}(t)$ to settle.

If data are collected for at least $\frac{1}{\bar{\eta}}$ seconds and the settling time is independent of the excitation signal, then the test time required for sequential mutlisine excitation does not depend on the number of inputs. That is, the system can be excited though all channels simultaneously.

\section{TWO-DEgREE-OF-FreEdom SPRING-MASS-DAMPER WITH CUBIC STIFFNESS}

Consider the two-degree-of-freedom spring-mass-damper

$$
M \ddot{q}+C \dot{q}+K(q)=b u,
$$

where $q \triangleq\left[\begin{array}{ll}q_{1} & q_{2}\end{array}\right]^{\mathrm{T}}$,

$$
\begin{aligned}
M & \triangleq\left[\begin{array}{ll}
m_{1} & \\
& m_{2}
\end{array}\right], \quad C \triangleq\left[\begin{array}{cc}
c_{1}+c_{2} & -c_{2} \\
-c_{2} & c_{2}
\end{array}\right], \\
K(q) & \triangleq\left[\begin{array}{c}
k_{1}\left(q_{1}\right)-k_{2}\left(q_{2}-q_{1}\right) \\
k_{2}\left(q_{2}-q_{1}\right)
\end{array}\right], \quad b \triangleq\left[\begin{array}{l}
0 \\
1
\end{array}\right] .
\end{aligned}
$$

Assume that the nonlinear stiffness $k_{1}(\cdot)$ and $k_{2}(\cdot)$ are given by the cubic stiffness terms $k_{1}(x) \triangleq \tilde{k}_{1} x+\hat{k}_{1} x^{3}$ and $k_{2}(x) \triangleq$ $\tilde{k}_{2} x+\hat{k}_{2} x^{3}$. The masses are $m_{1}=m_{2}=0.1 \mathrm{~kg}$. The damping coefficients are $c_{1}=0.1 \mathrm{~kg} / \mathrm{sec}$ and $c_{1}=0.05 \mathrm{~kg} / \mathrm{sec}$. The spring constants are $\tilde{k}_{1}=306 \mathrm{~kg} / \mathrm{sec}^{2}, \tilde{k}_{2}=20 \mathrm{~kg} / \mathrm{sec}^{2}, \hat{k}_{1}=550$ $\mathrm{kg} / \mathrm{sec}^{2} / \mathrm{m}^{2}$, and $\hat{k}_{2}=250 \mathrm{~kg} / \mathrm{sec}^{2} / \mathrm{m}^{2}$. The system (9.1)-(9.3) is linearizable about the origin, which is an asymptotically stable equilibrium. Let the output $y$ be the position of the first mass, that is $y=q_{1}$. The linearized system from input $u$ to output $y$ is $u=G(s) y$, where

$$
G(s) \triangleq \frac{5 s+2000}{\left(s^{2}+1.561 s+3273\right)\left(s^{2}+0.4394 s+187.0\right)} .
$$

The system has modes at approximately $2.18 \mathrm{~Hz}$ and $9.11 \mathrm{~Hz}$. Now let us consider excitation signals designed for frequency domain system identification over the frequency band $\omega_{\min }=0.1 \mathrm{~Hz}$ and $\omega_{\max }=25 \mathrm{~Hz}$ with a frequency spacing of $\eta=0.1 \mathrm{~Hz}$. The peak value of the excitation signal must be normalized to approximately $A=1.0$.

We consider the following three excitation signals.

(1) The single multisine given by (4.1) where $N=250$ and $A_{\mathrm{m}}=\frac{A}{\sqrt{N}}=\frac{1.0}{\sqrt{250}} \mathrm{~N}$.

(2) The sine dwell signal (5.1) where $N=250$ and $A_{\mathrm{s}, 1}, \ldots, A_{\mathrm{s}, N}=1.0 \mathrm{~N}$.

(3) The sequential multisine (7.1) where $M=8>\log _{2}\left(\frac{\omega_{\max }}{\omega_{\min }}\right)$ and, for $i=1, \ldots, M, A_{\mathrm{sm}, i}=\frac{A}{\sqrt{N_{i}}}$ where $N_{i}=$ $\frac{\bar{\omega}_{i+1}-\bar{\omega}_{i}}{\eta}$

To obtain equivalent signal-to-noise ratios, the single multisine excitation is averaged over $l_{\mathrm{m}}=N$ periods, for $k=1, \ldots, N$, the sine dwell excitations are averaged over $l_{\mathrm{s}, k}=\frac{\omega_{k}}{\eta}$ periods, and, for $i=1, \ldots, M$, the sequential multisine excitations are averaged over $l_{\mathrm{sm}, i}=N_{i}$ periods.

To illustrate the effects of nonlinearity induced harmonics, we consider the case where there is no noise on the input or the output. We excite the system (9.1)-(9.3) using the single multisine excitation. The system is allowed $\Delta t_{\mathrm{m}}=10 \mathrm{sec}$ to settle. Then data are collected for $l_{\mathrm{m}}=N=250$ periods of the single multisine, yielding $T_{\mathrm{m}}=2,510 \mathrm{sec}=41 \mathrm{~min} 50 \mathrm{sec}$. The transfer function (9.4) of the linearized system and the frequency response data obtain using the single multisine are shown in Figure 2. Note the distortions in the frequency response at frequencies
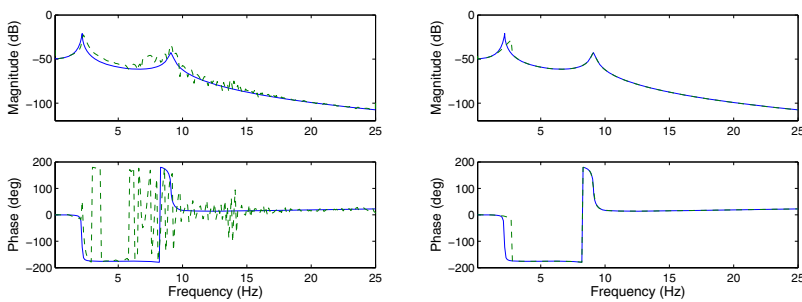

Fig. 2. Transfer function of the linearized system (solid) and the frequency response data obtain using the single multisine excitation (dashed).

Fig. 3. Transfer function of the linearized system (solid) and the frequency response data obtain using the sine dwell excitation (dashed).

higher than approximately $6 \mathrm{~Hz}$. Input-output data are collected for 2,500 sec at a sample rate of $500 \mathrm{~Hz}$. To obtain the frequency response data, we use Welch's averaged periodogram method, averaging 250 square windows of 5,000 data points to obtain 5,000 fast Fourier transform points.

Next, we excite the system (9.1)-(9.3) with the sine dwell signal. The system is allowed $\Delta t_{\mathrm{s}, 1}, \ldots, \Delta t_{\mathrm{s}, N}=10 \mathrm{sec}$ to settle at each frequency. For $k=1, \ldots, N$, we collect data for $l_{\mathrm{s}, k}=$ $\frac{\omega_{k}}{\eta}=k$ periods of the sinusoid $u_{\mathrm{s}, k}(t)$. Thus, $T_{\mathrm{s}}=5,000 \mathrm{sec}=$ $1 \mathrm{hr} 23 \mathrm{~min} 20 \mathrm{sec}$. The transfer function (9.4) of the linearized system and the frequency response data obtain using the sine dwell input are shown in Figure 3.

Finally, the system (9.1)-(9.3) is excited with the sequential multisine, and allowed $\Delta t_{\mathrm{ms}, 1}, \ldots, \Delta t_{\mathrm{ms}, M}=10 \mathrm{sec}$ to settle. For $i=1, \ldots, M$, we collect data for $l_{\mathrm{sm}, i}=N_{i}$ periods of $u_{\mathrm{sm}, i}(t)$, yielding $T_{\mathrm{sm}}=2,580 \mathrm{sec}=43 \mathrm{~min}$. The transfer function (9.4) of the linearized system and the frequency response data obtain using the sequential multisine are shown in Figure 4.

Figures 2-4 illustrate that the frequency response data obtained from the single multisine excitation provides the poorest approximation the linearized dynamics. Nonlinear distortions in the frequency response occur above $6 \mathrm{~Hz}$ and are a partial result of the third harmonic of the $2.18 \mathrm{~Hz}$ mode. The frequency response data obtained from the sine dwell excitation, shown in Figure 3, and the frequency response data obtained from the sequential multisine excitation, shown in Figure 4, both provide good approximations of the linearized dynamics. Both sets of frequency response data display some nonlinear distortion near the $2.18 \mathrm{~Hz}$ mode, but this distortion is not due to harmonic responses. Furthermore, if the input and output data obtained from the sine dwell and sequential multisine excitations were corrupted by the same noise sequence, then the associated noise levels of the two sets of frequency response data would be comparable. However, the sine dwell testing takes 40 minutes 20 seconds longer than the sequential multisine testing.

\section{Deployable Optical Telescope}

The Deployable Optical Telescope (DOT) is a space-traceable sparse-aperture telescope developed by the Air Force Research Laboratory $[7,8]$. The weight and volume restrictions imposed by launch vehicles are the main impediments to fielding large aperture space telescopes. To alleviate volume restrictions, DOT's primary mirror segments and secondary mirror tower are deployable. Specifically, the primary mirror segments rotate into place via hinges and lock with latches. The secondary mirror tower slides into place and locks with latches. 
A precise alignment of DOT's optical path is required to meet imaging requirements, and DOT requires active feedback control to reject image path disturbances caused by, for example, thermal variations and the motion of the spacecraft's reaction wheels. Each primary mirror segment is actuated with three degrees of freedom, namely tip, tilt, and piston. The bending modes of the secondary tower are controlled using a piezo patch bonded to the base of the tower. DOT uses laser metrology to provide opticalprecision measurements for feedback control. Specifically, the laser metrology system provides tip, tilt, and piston measurements for each of the three primary mirror segments. In sum, DOT has 10 control inputs and 9 feedback measurements.

High-fidelity linear dynamic models of DOT are required to design active feedback controllers that reject disturbances to DOT's optical path. Obtaining input-output data for system identification of DOT is difficult because system nonlinearities induce harmonic responses [8]. Sine dwell excitation signals could be used to obtain frequency response data of DOT's base tone responses, but this is very time consuming because performance objectives require frequency response data over a large frequency bandwidth (0 to $1000 \mathrm{~Hz}$ ), and DOT has densely spaced modes, requiring a fine frequency spacing. Therefore, the system identification process would be improved using the sequential multisine excitation signal.

We compare the frequency response data obtained from DOT when using single multisine, sine dwell, and sequential multisine excitation signals. DOT is excited through the first actuator on the first primary mirror segment and the piston position of the first primary mirror segment is measured. Thus, we obtain frequency response data from the first actuator of the first mirror to the piston position of the first mirror. To compare the exictation signals, frequency response data are obtained over the frequency band $\omega_{\min }=0.5 \mathrm{~Hz}$ to $\omega_{\max }=90 \mathrm{~Hz}$ with a frequency spacing of $\eta=0.5 \mathrm{~Hz}$. The peak value of the excitation signal must be normalized to approximately $A=0.02$. We consider the following three excitation signals.

(1) Single multisine given by (4.1) where $N=180$ and $A_{\mathrm{m}}=$ $\frac{A}{\sqrt{N}}=\frac{0.02}{\sqrt{180}} \mathrm{~N}$.

(2) Sine dwell signal (5.1) where $N=180$ and $A_{\mathrm{s}, 1}, \ldots, A_{\mathrm{s}, N}=0.02 \mathrm{~N}$.

(3) Sequential multisine (7.1) where $M=8>\log _{2}\left(\frac{\omega_{\max }}{\omega_{\min }}\right)$ and, for $i=1, \ldots, M, A_{\mathrm{sm}, i}=\frac{A}{\sqrt{N_{i}}}$ where $N_{i}=\frac{\bar{\omega}_{i+1}-\bar{\omega}_{i}}{\eta}$.

To obtain equivalent signal-to-noise ratios, the single multisine excitation is averaged over $l_{\mathrm{m}}=360$ periods, for $k=1, \ldots, N$, the sine dwell excitations are averaged over $l_{\mathrm{s}, k}=2 \frac{\omega_{k}}{\eta}$ periods, and, for $i=1, \ldots, M$, the sequential multisine excitations are averaged over $l_{\mathrm{sm}, i}=2 N_{i}$ periods.

For all excitation signals, the system is allowed $\Delta t_{\mathrm{m}}=$ $\Delta t_{\mathrm{s}, 1}, \ldots, \Delta t_{\mathrm{s}, N}=\Delta t_{\mathrm{ms}, 1}, \ldots, \Delta t_{\mathrm{ms}, M}=10 \mathrm{sec}$ to settle. Therefore, the single multisine, sine dwell, and sequential multisine test times are $T_{\mathrm{m}}=l_{\mathrm{m}} \frac{1}{\eta}+\Delta t_{\mathrm{m}}=730 \mathrm{sec}=12 \min 10 \mathrm{sec}$, $T_{\mathrm{s}}=\sum_{k=1}^{N} l_{\mathrm{s}, k} \frac{1}{\omega_{k}}+\Delta t_{\mathrm{s}, k}=2,520 \mathrm{sec}=42 \mathrm{~min}$, and $T_{\mathrm{sm}}=$ $\sum_{i=1}^{M} l_{\mathrm{sm}, i} \frac{1}{\eta}+\Delta t_{\mathrm{sm}, i}=800 \mathrm{sec}=13 \mathrm{~min} 20 \mathrm{sec}$, respectively. Sine dwell testing takes significantly longer than single multisine or sequential multisine testing, but single multisine testing cannot be used because of the harmonic distortion that results from system nonlinearities.

The data obtained from sine dwell excitation are used to calculate the total harmonic distortion of DOT's dynamics from the first actuator of the first mirror to the piston position of the first mirror. The total harmonic distortion is the ratio of output
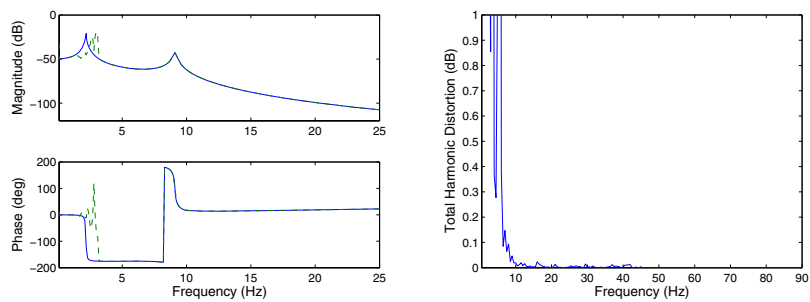

Fig. 4. Transfer function of the lin- Fig. 5. Total harmonic distortion earized system (solid) and the fre- from the control on the first priquency response data obtain using mary mirror segment to the piston the sequential multisine excitation response of the first primary mirror (dashed). segment.
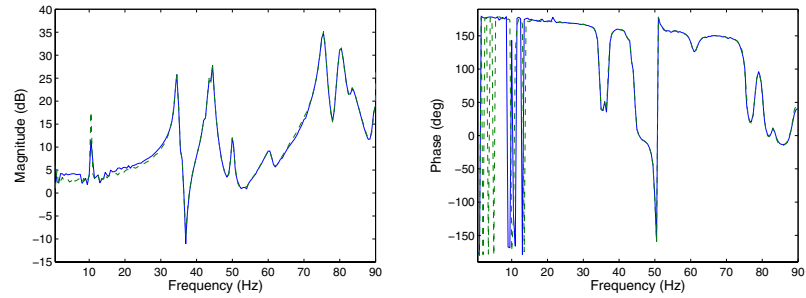

Fig. 6. The magnitude (left) and phase (right) of the frequency response data obtain using the sequential multisine excitation (solid) and the single multisine excitation (dashed).

power at all superharmonic frequencies to the output power at the base frequency. Figure 5 shows that total harmonic distortion of DOT's dynamics from the first actuator of the first mirror to the piston position of the first mirror. Figure 5 demonstrates that there is significant harmonic distortion due to base frequencies up to 10 $\mathrm{Hz}$ and lower levels of distortion due to higher base frequencies.

Figure 6 show the magnitude and the phase of the frequency response data obtained from the single multisine and sequential multisine excitation signals. The two frequency response differ most significantly between 0.5 and $30 \mathrm{~Hz}$. This difference can be attributed to the harmonic distortion that is present in the single multisine data but is not present in the sequential multisine data.

\section{REFERENCES}

[1] M. A. Schroeder, "Synthesis of low peak-factor signals and binary sequences of low autocorrelation," IEEE Trans. Info. Theory, vol. 16, pp. 85-89, 1970.

[2] M. W. Braun, R. Ortiz-Mojica, and D. E. Rivera, "Application of minimum crest factor multisinusoidal signals for "plant-friendly" identification of nonlinear process systems," Contr. Eng. Practice, vol. 10, pp. 301-313, 2002.

[3] H. A. Barker and K. R. Godfrey, "System identification with multilevel periodic perturbation signals," Contr. Eng. Practice, vol. 7, pp. 717-726, 1999.

[4] J. Schoukens, R. M. Pintelon, and Y. J. Rolain, "Broadband versus stepped sine frf measurements," IEEE Trans. Instr. and Meas., vol. 49, pp. 275-278, 2000.

[5] R. Pintelon and J. Schoukens, System Identification: A Frequency Domain Approach. Piscataway, NJ: IEEE Press, 2001.

[6] P. Guillaume, J. Schoukens, R. Pintelon, and I. Kollár, "Crest-factor minimization using nonlinear chebyshev approximation methods," IEEE Trans. Instr. and Meas., vol. 40, pp. 982-989, 1991.

[7] K. N. Schrader, R. H. Fetner, S. F. Griffin, and R. S. Erwin, "Development of a sparse-aperture testbed for optomechanical control of spacedeployable structures," in Proc. SPIE Vol. 4849: Highly Innovative Space Telescope Concepts, Waikoloa, HI, Dec 2002, pp. 384-395.

[8] S. L. Lacy, V. Babuška, K. N. Schrader, and R. Fuentes, "System identification of space structures," in Proc. Amer. Contr. Conf., Portland, OR, June 2005, pp. 2335-2340. 